\title{
PERCEPÇÃO DE PAIS/CUIDADORES SOBRE A ETIOLOGIA DO POSSÍVEL BRUXISMO DO SONO EM ESCOLARES
}

\section{PARENTS AND CAREGIVERS' KNOWLEDGE ABOUT POSSIBLE SLEEP BRUXISM IN SCHOOLCHILDREN}

\author{
Maria Isabel Braz Vieira ${ }^{1}$ \\ Maria Letícia Ramos Jorge ${ }^{2}$ \\ Antonio Lopes Beserra Neto ${ }^{3}$ \\ Lívia Pereira Brocos Pires ${ }^{4}$ \\ Ankilma do Nascimento Andrade Feitosa ${ }^{5}$ \\ Clarissa Lopes Drumond ${ }^{6}$
}

RESUMO: OBJETIVO: Avaliar associação entre a percepção dos pais/cuidadores sobre a etiologia do bruxismo do sono e o possível bruxismo do sono em seus filhos. MÉTODOS: Um estudo transversal realizado com amostra de 439 escolares de 8 a 10 anos de idade de escolas públicas e particulares da cidade de Diamantina, Minas Gerais e seus pais e cuidadores. Os pais/cuidadores responderam um questionário pré-estruturado com informações sobre as crianças:som característico de ranger os dentes durante o sono, conhecimento dos pais/cuidadores sobre a causa de bruxismo do sono e fatores sociodemográficos (idade da criança, número de filhos, ordem do nascimento da criança, escolaridade dos pais/cuidadores, renda mensal familiar, duração da renda). O diagnóstico de bruxismo do sono foi baseado no relato dos pais (possível bruxismo do sono) de acordo como o consenso internacional. A análise descritiva e inferencial (frequência absoluta e relativa dos dados e teste qui-quadrado $(P \leq 0,05))$ foram realizadas. RESULTADOS: $A$ prevalência de possível bruxismo do sono foi de $40,1 \%(n=176)$. Um total de $54,7 \%$ $(n=240)$ das crianças eram do sexo feminino e $55,1 \%(n=242)$ dos pais/cuidadores apresentaram escolaridade menor que 8 anos de estudo. Além disso, 38,2\% $(n=71)$ dos pais/cuidadores relataram que não tinham conhecimento da etiologia do ranger os dentes, enquanto $29,0 \%$ ( $n=54)$ dos pais/cuidadores relataram que a etiologia do ranger os dentes dos seus filhos era devida à presença de vermes e $15,1 \%(n=28)$

\footnotetext{
${ }^{1}$ Graduada em Odontologia. Faculdade Santa Maria, Paraíba, Brasil.

2 Doutora em Odontologia, Docente do Departamento de Odontopediatria, Faculdade de Odontologia, Universidade Federal dos Vales do Jequitinhonha e Mucuri, Minas Gerais, Brasil.

${ }^{3}$ Graduado em Odontologia. Faculdade Santa Maria, Paraíba, Brasil.

${ }^{4}$ Mestre em Odontologia. Docente da Faculdade Santa Maria, Paraíba, Brasil.

${ }^{5}$ Doutora em Ciências da Saúde, Docente da Faculdade Santa Maria, Paraíba, Brasil.

${ }^{6}$ Doutora em Odontologia, Docente da Faculdade Santa Maria, Paraíba, Brasil.
} 
acreditavam que era devido à fatores psicossociais (ansiedade, estresse). A falta de conhecimento de pais/cuidadores sobre a etiologia do bruxismo do sono foi associada com o relato de bruxismo do sono do seu filho (possível bruxismo do sono) $(p=0,043)$. Não houve associação estatisticamente significativa nas demais variáveis independente com o possível bruxismo do sono. CONCLUSÃO: A falta de conhecimento dos pais / cuidadores quanto à etiologia do ranger os dentes foi associada à presença de PSB.A maioria dos pais / cuidadores da presente amostra parece desconhecer a etiologia do ranger os dentes de seus filhos durante o sono.

Palavras chave: Bruxismo do Sono. Criança. Epidemiologia.

ABSTRACT: OBJECTIVE: To evaluate the association between Self reported knowledge of parents / guardian about the etiology and presence of possible sleep bruxism (PSB) among their children. Methods: A cross-sectional study was conducted with a sample of 439 students aged 8 to 10 years old from public and private schools in the city of Diamantina, Minas Gerais, Brazil and their parents and caregivers. Parents /caregivers answered a pre-structured questionnaire with the following information about the children: identification of peculiar sound of teeth grinding during sleep, knowledge of parents/ caregivers about the etiology of sleep bruxism and sociodemographic factors (age of the child, number of children in the family, order of child birth, parents/caregivers level of education, monthly family income, duration of income). The diagnosis of sleep bruxism was based on the parentsl caregivers' report (PSB) according to the international consensus. Descriptive and inferential analysis (absolute and relative frequency of data and chisquare test, $p \leq 0.05$ ) were performed. Results: The prevalence of PSB was $40.1 \%$ ( $n$ $=176)$. A total of $54.7 \%(n=240)$ of the children were female and $55.1 \%(n=242)$ of the parents / caregivers had less than 8 years of schooling. In addition, $38.2 \%(n=$ 71) of parents / caregivers reported that they were unaware of the etiology of teeth grinding, while $29.0 \%(n=54)$ of parents / caregivers reported that the etiology of teeth grinding of their children was due to the presence of intestinal parasitic infections and $15.1 \%(n=28)$ believed that it was due to psychosocial factors (anxiety, stress). The lack of knowledge of parents / caregivers about the etiology of sleep bruxism was associated with thereport of child'ssleep bruxism (PSB) ( $p=0.043)$. There was no statistically significant association with respect to other independent variables and PSB. Conclusion: The lack of knowledge of parents/ caregivers related to the etiology of teeth grinding was significantly associated to the presence of PSB.Most of the parents / caregivers of the present sample seem to be unaware of the etiology of their children's teeth grinding during sleep.

Keywords: Sleep Bruxism. Child. Epidemiology. 


\section{INTRODUÇÃO}

O bruxismo do sono (BS) é caracterizado por movimentos rítmicos e não rítmicos da musculatura mastigatória. Estes movimentos estão associados com o ranger e/ou apertar dos dentes, bem como o apoio ou empurrão dos mesmos durante o sono (LOBBEZOO et al., 2018).

A prevalência de BS entre crianças apresenta uma alta variabilidade, devido às diferentes metodologias de diagnóstico e faixa etária avaliada. De acordo com dados da literatura, em escolaresvaria de 3,5 a 40,6\% (GONÇALVES et al., 2009; MANFREDINI et al., 2013; SERRA NEGRA et al., 2013; THOMAZ; CANGUSSU; ASSIS, 2013).

Diversos são os fatores que podem influenciar na prevalência de BS em crianças, como bullying escolar (FULGENCIO et al., 2016): distúrbios respiratórios (rinite e sinusite) (DRUMONDet al., 2018), ansiedade (GUO, HUAQ, 2018) estresse(DRUMONDet al., 2020), traços de personalidade (SERRA-NEGRA et al.,2012), transtorno de déficit de atenção e hiperatividade (MOTA-VELOSO et al., 2017), alta escolaridade materna (DRUMOND et al., 2017), vulnerabilidade familiar (SERRA-NEGRA et al. ,2013). A dinâmica familiar, atitudes dos pais na imposição de tarefas domésticas, autoridade paterna e materna também estão associados ao bruxismo do sono em crianças (DRUMOND et al., 2020; SERRA-NEGRA et al., 2013).

Diferentes abordagens multidisciplinares e teóricas sobre as possíveis causas do bruxismo apontam etiologia multifatorial que pode incluir fatores locais, sistêmicos, psicológicos, ocupacionais e hereditários(COSTA et al., 2017).Crianças com bruxismo podem apresentar outras condições relacionadas ao sono, algumas condições como solilóquio, sonambulismo, fazer xixi na cama, roncar e pesadelos (KHATWA; KOTHARE. 2013).Também é possível que situações de desestruturação e agitação social e adversidades durante o dia possam ocasionar um sono agitado à 
noite, promovendo alterações funcionais nos níveis de contrações musculares(DAL FABRO; CHAVES JUNIOR, 2012; KHATWA; KOTHARE. 2013).

O BS pode levara efeitos deletérios como fadiga, dore hipertrofia muscular, dor temporomandibular, cefaleia, desgastes dentários e impacto negativo na qualidade de vida do indivíduo (CARRA et al., 2011; FERNANDES et al., 2013; GHAFOURNIA; TEHRANI, 2012; LOBBEZOO et al., 2012; LOBBEZOO et al., 2017; PAESANI, 2010; SERRA-NEGRA et al., 2012).

Estudos evidenciaram que os pais/cuidadores desconhecem a etiologia do BS, associando essa condição a ser de origem mística, religiosa ou pela presença de parasitas (SERRA-NEGRA et al.,2013; SILVA et al., 2016).

O diagnóstico precoce do BS ajuda a fornecer uma perspectiva de controle, evita danos aos componentes do sistema mastigatório (SERRA-NEGRA et al., 2013). Logo, nota-se que o conhecimento dos pais/responsáveis acerca da etiologia do BS é de grande relevância para o direcionamento da prevenção e gerenciamento dessa condição (LOBBEZOO et al., 2018).

Sabendo que o conhecimento pelos pais sobre essa condição bucal é essencial para direcioná-los a procuraratendimento à criança, realizar um estudo que explore esta temática em uma população de região diferente das que foram estudadas até o momento é importante. Além disso, este estudo poderá colaborar para o desenvolvimento de um modelo teórico que estruture as variáveis para realização de futuros estudos com desenhos mais elaborados. Consequentemente, será importante para traçar possíveis estratégias de educação em saúde voltadas para os pais/cuidadores sobre a etiologia do BS.

Dessa forma, o objetivo do presente estudo foi avaliar associação entre a percepção dos pais/cuidadores sobre a etiologia do bruxismo do sono e o possível bruxismo do sono em seus filhos. 


\section{MATERIAIS E MÉTODOS}

O presente estudo foi aprovado pelo Comitê de Ética em Pesquisa da Universidade Federal dos Vales do Jequitinhonha e Mucuri (Brasil), no 370.291. Os pais/responsáveis pela criança foram convidados a assinar o Termo de Consentimento Livre e Esclarecido (TCLE).

Este estudo transversal foi realizado, no ano de 2013, com crianças escolares de 8 a 10 anos de idade,e seus pais/cuidadores recrutados de forma aleatória nas escolas públicas e particularesda cidade de Diamantina, Minas Gerais. Os escolares com dentição permanente, submetidos a tratamento ortodôntico, alterações neurológicas, e os pais respondentes que não dormiam na mesma casa que a criança e não apresentavam domínio de leitura foram excluídos do estudo.

O cálculo da amostra foi realizado no programa Epi Info program (version 7). Foi consideradopara o cálculo o nível de significância de 95\%, erro de estimativa de $5 \%$. A prevalência de $23,1 \%$ do conhecimento dos pais/cuidadores sobre bruxismo do sono foi adotada (PRADO et al., 2019). Uma amostra de 384 escolares foi necessária. Foi adicionada $50 \%$ à amostra, a fim de compensar possíveis perdas. Além disso, o efeito deff de 1,2 foi computado, totalizando a amostra de 491 crianças em idade escolar. A distribuição da amostra foi realizada a partir do sorteio das escolas e, em seguida, das salas de aula. Então, quatro escolas públicas e uma particular participaram da pesquisa.

Coleta de dados

Os pais/cuidadores responderam o questionário pré-estruturado autoadministrado. No questionário pré-estruturado, contém informações sobre a identificação e saúde geral da criança, se seu filho rangia os dentes durante o sono, sobre apercepção dos pais/cuidadores sobre a etiologia do bruxismo do sono, 
aspectos sociodemográficos, idade da criança, número de filhos, ordem de nascimento da criança, escolaridade dos pais/cuidadores, renda mensal familiar e duração da renda.

O diagnóstico do possível bruxismo do sono (PBS) da criança foi obtido a partir do relato dos pais/cuidadores de acordo com o último consenso de BS.Os pais/cuidadores foram orientados a acompanhar o sono da criança por 2 semanas para observar a presença de ranger de dentes dos seus filhos durante o sono (LOBBEZOO et al., 2018).

A equipe de pesquisa foi composta por um cirurgião-dentista eum auxiliar para organização dentro da escola e a distribuição dos questionários. Todos os avaliadores passaram por um treinamento sobre o conceito e etiologia do bruxismo do sono, além da forma de abordagem aos pais e seus filhos durante a coleta de dados.

Após a realização do treinamento, foi realizado um estudo piloto com $10 \%$ da amostra, com o propósito de aprovar a metodologia proposta. Previamente ao estudo piloto, foi feito o teste reteste do questionário pré-estruturado.

\section{RESULTADOS}

Um total de 439 crianças (9,15 $\pm 0,83$ anos) juntamente com seus pais/cuidadores participaram da presente pesquisa, com uma taxa de resposta de $89,4 \%$. O motivo das perdas foipor ter questionários incompletos. A prevalência do PBS foi de 40,1\% $(n=176)$.

Das 439 crianças que participaram do estudo, 54,7\% $(n=240)$ eram do sexo feminino. Os pais/cuidadores com escolaridade menor ou igual a 8 anos de estudo corresponderam a $55,1 \%(n=242)$, e a renda mensal familiar de dois ou mais de dois salários mínimos, de acordo com o salário mínimo brasileiro no ano de 2013 $(R \$ 678,00)$, foi de $52,2 \%(n=229)$. Os pais/cuidadores relataram que $40,1 \%$ das crianças rangiam dente durante o sono (tabela 1 ). 
Em relação à etiologia do ranger de dentes durante o sono da criança, $38,2 \%$ $(n=71)$ dos pais/cuidadores declararam que não tinham conhecimento das causas dessa condição, 29,0\% ( $n=54)$ pais/cuidadores relacionaram o ranger de dentes dos seus filhos com a presença de verme e 15,1\% ( $n=28)$ com fatores psicossociais (Tabela 1).

Tabela 1. Distribuição de frequência do possível bruxismo do sono nas crianças, etiologia do mesmo segundo relato dos pais/cuidadores e fatores sociodemográficos. Diamantina, Minas Gerais, Brasil, 2013 ( $n=439)$.

\begin{tabular}{lc}
\hline Variáveis & $\mathbf{N}(\%)$ \\
\hline Variável dependente & \\
Possível bruxismo do sono & $263(59,9)$ \\
$\quad$ Ausente & $176(40,1)$ \\
Presente & \\
Variáveis independentes & \\
Sexo & $199(45,3)$ \\
$\quad$ Masculino & $240(54,7)$ \\
Feminino & \\
Idade & $251(57,2)$ \\
8-9 anos & $188(42,8)$ \\
10 anos & \\
Escolaridade dos pais/cuidadores & $197(44,9)$ \\
$\geq 8$ anos de estudo & $242(55,1)$ \\
$\leq 8$ anos de estudo & \\
Renda mensal familiar & $229(52,2)$ \\
2 ou mais salários mínimos & $210(47,8)$ \\
Menos de 2 salários mínimos & \\
Duração da renda & $278(63,3)$ \\
1 mês ou mais & $161(36,7)$ \\
Menos de 1 mês & $71(38,2)$ \\
Etiologia do bruxismo do sono segundo os pais/cuidadores \\
Não sabe & $54(29,0)$ \\
Verme & $28(15,1)$ \\
Fatores psicossociais & $5(1,1)$ \\
Alterações das vias aéreas & $4(0,9)$ \\
Posição dentária & $25(13,4)$ \\
Bruxismo &
\end{tabular}

$\mathrm{Na}$ análise bivariada, verificou a associação estatisticamente significativa entrepossível bruxismo do sono e pais/cuidadores que relataram não saberem a etiologia do ranger de dentes durante o sono de suas crianças $(p=0,043)$. Não houve 
associação estatisticamente significativa com as demais variáveis independentes com o possível bruxismo do sono (Tabela 2).

Tabela 2. Associação entre possível bruxismo do sono e a percepção dos pais/cuidadores quanto à etiologia do mesmo em suas crianças. Diamantina, Minas Gerias, Brasil, 2013.

\begin{tabular}{|c|c|c|c|c|}
\hline \multirow{2}{*}{$\begin{array}{l}\text { Etiologia do possível } \\
\text { bruxismo do sono das } \\
\text { crianças segundo os } \\
\text { pais/cuidadores }\end{array}$} & \multicolumn{2}{|c|}{$\begin{array}{c}\text { Possível bruxismo do } \\
\text { sono }\end{array}$} & \multirow{2}{*}{ Total } & \multirow{2}{*}{$p$-value ** } \\
\hline & $\begin{array}{c}\text { Ausente } \\
\text { n (\%) }\end{array}$ & $\begin{array}{c}\text { Presente } \\
\text { n (\%) }\end{array}$ & & \\
\hline $\begin{array}{l}\text { Conhecimento da causa } \\
\text { de BS } \\
\text { Relatam saber a causa } \\
\text { Não relatam saber a causa } \\
\text { Verme }\end{array}$ & $\begin{array}{c}7(6,1) \\
11(15,5)\end{array}$ & $\begin{array}{l}108(93,9) \\
60(84,5)\end{array}$ & $\begin{array}{l}115(100 \%) \\
71(100 \%)\end{array}$ & 0,043 \\
\hline $\begin{array}{l}\text { Não } \\
\text { Sim } \\
\text { Fatores psicossociais }\end{array}$ & $\begin{array}{c}15(11,4) \\
3(5,6)\end{array}$ & $\begin{array}{l}117(88,6) \\
51(94,4)\end{array}$ & $\begin{array}{l}132(100 \%) \\
54(100 \%)\end{array}$ & 0,283 \\
\hline $\begin{array}{l}\text { Não } \\
\text { Sim }\end{array}$ & $\begin{array}{c}18(11,4) \\
0(0)\end{array}$ & $\begin{array}{c}140(88,6) \\
28(100)\end{array}$ & $\begin{array}{l}158(100 \%) \\
28(100 \%)\end{array}$ & 0,08 \\
\hline $\begin{array}{l}\text { Vias aéreas respiratórias } \\
\text { Não } \\
\text { Sim }\end{array}$ & $\begin{array}{c}17(9,4) \\
1(20)\end{array}$ & $\begin{array}{c}164(90,6) \\
4(80)\end{array}$ & $\begin{array}{c}181(100 \%) \\
5(100 \%)\end{array}$ & 0,402 \\
\hline $\begin{array}{l}\text { Posição de dentes } \\
\text { Não } \\
\text { Sim }\end{array}$ & $\begin{array}{c}18(9,9) \\
0(0)\end{array}$ & $\begin{array}{c}164(90,1) \\
4(100)\end{array}$ & $\begin{array}{c}182(100 \%) \\
4(100 \%)\end{array}$ & 1,000 \\
\hline $\begin{array}{l}\text { Bruxismo do sono } \\
\text { Não } \\
\text { Sim }\end{array}$ & $\begin{array}{c}16(9,9) \\
2(8)\end{array}$ & $\begin{array}{c}145(90,1) \\
23(92)\end{array}$ & $\begin{array}{c}161(100 \%) \\
25(100 \%)\end{array}$ & 1,000 \\
\hline
\end{tabular}

\section{DISCUSSÃO}

Devido aos diversos métodos de avaliação e instrumentos utilizados nos estudos, é necessário cautela ao comparar os resultados da presente investigação com os demais trabalhos encontrados na literatura.

No presente estudo, a prevalência de possível bruxismo do sono foi alta.Outro estudo também corrobora com esse achado (PRADO et al., 2019). Deve-se 
considerar que essa prevalência pode ter sido influenciada pela idade da criança, bem como o tipo diagnóstico para bruxismo do sono empregado (LOBBEZOO et al., 2018).Para estapesquisa, foi adotado o relato dos pais/cuidadores (possível bruxismo do sono).

De acordo com os resultados encontrados, verificou a associação entre possível bruxismo do sono e pais/cuidadores que relataram não saberem a etiologia do ranger de dentes durante o sono de suas crianças.A etiologia do bruxismo do sono parece não ser clara entre os pais/cuidadores.Estes achados corroboram outras pesquisas (CALDERAN et al., 2014; CARDOSO et al., 2011; SILVA et al., 2016; PRADO et al., 2019). Esses dados, mais uma vez, trazem a necessidade da divulgação de informações a respeito do BS.

Isso serve de alerta, pois a família é a grande responsável pela detecção dessa condição.São eles que reconhecem os sons gerados pelo ranger os dentes durante o sono da criança.Resultados de estudos anteriores sugeriram que o bruxismo do sono na idade escolar é comum e que pode estar associado a diversos fatores etiológicos, como sinusite, rinite, estresse, tarefas domésticas, traço de personalidade e péssima qualidade do sono (DRUMOND et al., 2018; DRUMOND et al., 2020; SERRA-NEGRA et al., 2012; SOARES et al., 2020).

Não houve associação entre o relato dos pais/cuidadores de que o ranger os dentes dos seus filhos esteja relacionado com a presença de verme e com o possível bruxismo do sono. Apesar disso, há considerável parcela de pais/cuidadores acreditaram nessa teoria do ranger os dentes durante o sono dos seus filhos com presença de vermes. Outros estudos realizados no Brasil alertam para a necessidade de discussão e esclarecimento aos pais/cuidadores acerca dos mitos e tabus preexistentes no senso comum e conhecimento popular acerca das causas do bruxismo (BONACINA et al., 2020).

No presente estudo não houve associação entre o conhecimento sobre a etiologia do BS pelos pais/cuidadores com fatores psicossociais. Porém, há um número significativo de pais/cuidadores que acreditavam que o bruxismo do sono provém de problemas emocionais. A literatura tem mostrado a associação entre bruxismo do sono, estresse e ansiedade. Crianças com estresse tiveram maior 
chance de apresentarem bruxismo do sono em relação às crianças sem estresse (DRUMOND et al., 2020; GUO, HUAQ, 2018).

Além disso, aqueles pais que conheciam a etiologia do bruxismo do sono neste estudo foram poucos e essa variável não apresentou associação com o PBS.

A necessidade da desmistificação das crenças é necessária, visto que podem influenciar no tipo de tratamento procurado pelo paciente (SERRA-NEGRA et al., 2013).

Os pais/cuidadores podem procurar médicos em vez de dentistas. O pediatra geralmente costuma ser o primeiro profissional a estabelecer contato com uma criança, tendo papel importante no diagnóstico do BS (KELLY, MCCARTHY, SAHM, 2014). Assim, é importante destacar que deve haver uma abordagem multidisciplinar entre os profissionais de saúde (SERRA-NEGRA et al., 2013).

O presente estudo apresenta algumas limitações. O desenho de estudo transversal não permite verificar a relação de causa e efeito entre as variáveis investigadas com o desfecho. Além disso, o tipo de diagnóstico adotado para BS foi o relato dos pais/cuidadores. Esta é uma opção de diagnóstico estabelecida pelo último consenso internacional (LOBBEZOO et al., 2018) e ser o diagnóstico viável para estudos epidemiológicos que envolvem uma amostra representativa. Porém,o exame de polissonografia é considerado padrão-ouro para o diagnóstico de bruxismo do sono (RAPHAEL et al., 2015). No presente estudo, não foi utilizado esse exame, pois o custo e a complexidade de execução não possibilitam a sua realização em grande quantidade de crianças. Sendo isto, portanto, um fator limitante em estudos epidemiológicos com grande amostra.

Os dados dessa investigação norteiam para a elaboração de estratégias eficazes de prevenção e promoção de saúdea partir da informação correta à população (REIS et al., 2010). Além disso, enfatizam a importância do desenvolvimento de medidas de educação em saúde aos pais/cuidadores sobre a etiologia do bruxismo do sono em crianças, visto que o mesmo apresenta uma etiologia multifatorial e complexa. 


\section{CONCLUSÃO}

Em conclusão, houve associação entre pais/cuidadores que relataram não saber a etiologia do ranger os dentes durante o sono dos seus filhos com o possível bruxismo do sono. Os pais/cuidadores parecem desconhecer a etiologia do ranger os dentes dos seus filhos durante o sono.

\section{AGRADECIMENTOS}

Este estudo foi apoiado pela Coordenação de Aperfeiçoamento de Pessoal de Nível Superior (CAPES) e pelo CNPq. 


\section{REFERÊNCIAS BIBLIOGRÁFICAS}

BONACINA, CF. et al. Associação entre bruxismo do sono e personalidade da criança sob a percepção dos pais/cuidadores. Revista Saúde-UNG-Ser, 2020; 14: 16-22.

CALDERAN, MF. et al. Fatores etiológicos do Bruxismo do Sono: revisão de Literatura. Revista de Odontologia Da Universidade Cidade de São Paulo. 2014; 26: 243-249.

CARDOSO, F. et al.The Dental Aesthetic Index and Dental Health Component Of The Index Of Orthodontic Treatment Need as Tools in Epidimiological Studies. Internacional Journal of Environmental Research and Public Health. 2011; 8, (8): 3277-3286.

CARRA, M. et al. Prevalence And Risk Factors Of Sleep Bruxism And Wake-Time Tooth Clenching In A 7- To 17-Yr-Old Population. Eur J Oral Sci, 119(5):386-394, 2011.

CLEMENTINO, MA. et al.Siqueira MB , Serra -Negra JM , Paiva SM , Granville -Garcia AF. The prevalence of sleep bruxism and associated factors in children: a report by parents. Eur Arch Paediatr Dent 2017;18(6):399 -404.

COSTA, ARO. et al. Prevalência e fatores associados ao bruxismo em universitários: um estudo transversal piloto. Revista Brasileira de Odontologia. 2017; 74 (2): 120.

DAL FABRO, C; CHAVES JÚNIOR, CM. Bruxismo do sono [Sleep bruxism]. In: Dal Fabro C, Chaves Júnior $C M$, Tufik $S$, (eds.) The dentistry in sleep medice. 1st ed. Maringá: Dental Press; 2012. p. 345-74.

DRUMOND, CL, et al. of probable sleep bruxism and associated factors in brazilian schoolchildren. International Journal of Paediatric Dentistry.2018;29 (2): 1-7.

DRUMOND, CL. et al. Respiratory disorders and the prevalence of sleep bruxism among schoolchildren aged 8 to 11 years. Sleep\& Breath = Schlaf\& Atmung. 2017; 21, (1): 203-208.

DRUMOND, CL. et al. Do Family Functioning and Mothers and Children's Stress Increase the Odds of Probable Sleep Bruxism Among Schoolchildren? A Case Control Study. Clinical Oral InvestigationsI. 2020; 24 (2): 1025-1033.

FERNANDES G. et al. Tem-Poromandibular Disorders, Sleep Bruxism, And Primary Headaches Are Mutually Associated. J Orofac Pain. 27: 14-20, 2013.

FULGENCIO, LB. et al. Diagnosis of sleep bruxism can assist in the detection of cases of verbal school bullying and measure the life satisfaction of adolescents. International Journal Paediatric Dentistry. 2016; 27 (4): 293-301.

GHAFOURNIA, M.; TEHRANI, M. H. Relationship Between Bruxism And Malocclusion Among Preschool Children In Isfahan. J Dent Res Dent Clin Dent Prospects. 2012 6(4):138-142, 2012.

GUO, HUAQ. The risk factors related to bruxism in children: A systematic review and metaanalysis. Arch Oral Biol. 2018; 86: 18-34.

KELLY M; MCCARTHY S; SAHM LJ. Knowledge, attitudes and beliefs of patients and carers regarding medication adherence: a review of qualitative literature. Eur $\mathbf{J}$ Clin Pharmacol. 2014;70: 1423 - 1431. 
KHATWA U; KOTHARE SV. Sleep bruxism. In: Kothare SV, Ivanenko A, (eds.) Parasomniasclinical characteristics and treatment. 1st ed. New York: Springer; 2013. p. 281-92.

LOBBEZOO F. et al. Bruxism Defined and Graded: An International Consensus. J Oral Rehabil, v. 40, p. $2-4,2013$.

LOBBEZOO F. et al. Sleep Bruxism: Diagnostic Considerations. P. 1427-1434. In Kryger M, Roth T, Dement W (Editors) Principles And Practice Of Sleep Medicine. Philadelphia: Elsevier, 2017.

LOBBEZOO, F. et al. International Consensus on the Assessment of Bruxism: Report of a Work in Progress. Journal of Oral Rehabilitation. 2018; 5: 837-844.

MANFREDINI, D. et al .Prevalence of sleep Bruxism in Children: a Systematic Review of the Literature. Journal of Oral Rehabilitation. 2013; 40 (8): 631-642.

MOTA-VELOSO, I. et al. Effects of Attention Deficit Hyperactuvity Disorder Signs and SocioEconomic Status on Sleep Bruxism and Tooth Wear Among Schoolchidren: Structural Equation Modelling Approach. Internacional Journal of Paediatric Dentistry. 2017; 27 (6): 523-531.

PRADO, IM. et al., Knowledge of parents/caregivers about the sleep bruxism of their children from all five Brazilian regions: A multicenter study.Int J Paediatr Dent. 2019; 29 (4): 507-523.

RAPHAEL, KG. et al. Validity of Self- reported sleep bruxism among myofascial temporomandibular discord patients and controls. Journal of Oral Rehabilitation. 2015; 42 (10): 751-758.

REIS, DM. et al. Educação em saúde como estratégia de promoção de saúde bucal em gestantes. Ciência \& Saúde Coletiva, 2010, 15: 269-276.

SERRA-NEGRA JM. et al.Relationship between tasks performed, personality traits, and sleep bruxism in Brazilian school children--a population-based cross-sectional study. PLoS One. 2013; 8 (11): 1-6.

SERRA-NEGRA, JM. et al .Association Among Stress, Personality Traits, and Sleep Bruxism in Children. Pediatric Dentistry. 2012; 34: 30-34.

SERRA-NEGRA, JM. et al. Evaluation of parents/guardian knowledge about the bruxism of their children: family knowledge of bruxism. J Indian Soc Pedod Prev Dent. 2013; 31: 153-8.

SILVA, CT.et al.Knowledge of parents/guardians about nocturnal bruxism in children and adolescentes. The Journal of Craniomandibular \& Sleep Practice. 2016; 223-227.

SOARES, JP. et al. Association of gender, oral habits, and poor sleep quality with possible sleep bruxism in schoolchildren. Braz. Oral Res.2020; 34: 1807-3107. 